\title{
A Review of Space-Time Block Codes from Real and Complex Orthogonal Designs
}

\author{
Swapnil T. Patil \\ Department of Electronics and Telecommunication \\ Sinhgad Academy of Engineering, Kondhwa \\ Pune, India
}

\author{
Pratap N. Shinde, Ph.D \\ Department of Electronics and Telecommunication \\ Sinhgad Academy of Engineering, Kondhwa \\ Pune, India
}

\begin{abstract}
This paper discusses the fundamental concepts behind the real and complex orthogonal designs for space-time block coding used in wireless communication systems. Generalized real orthogonal design and complex orthogonal designs are discussed in this paper. Also a brief survey of existing orthogonal designs for space-time block coding is put forward in this paper.
\end{abstract}

\section{General Terms}

Wireless communication, space-time block coding.

\section{Keywords}

Space-time block coding, real orthogonal design, generalized complex orthogonal design.

\section{INTRODUCTION}

Space time block codes from orthogonal designs can be divided into two parts. First part takes into consideration real orthogonal designs for real signal constellation. For example phase amplitude modulation (PAM). Second part takes into consideration complex orthogonal designs for complex constellation examples is phase shift keying (PSK). The space-time block code (STBC) from complex orthogonal designs is important to achieve high data rates using Quadrature amplitude modulation (QAM) signal constellation in broadband wireless communication [1]. The real orthogonal designs are based on compositions of quadratic forms which are well developed in the form of mathematics literature [2-3]. Ganesan and Stoica [4] revisited this scheme to achieve maximum signal to noise ratio (SNR). Unlike STBC from real orthogonal designs, complex orthogonal designs are not well understood.

Hurwitz-Radon theory [5-7] describes that for any transmit antenna's rate one can be reached by real orthogonal designs. Tarokh, Jafarkhani and Calderbank [3] described that for STBC from real orthogonal designs and complex orthogonal designs their rates cannot be greater than one. Recently Liang Xian and Huaping Liu [8] explained that rate one for complex orthogonal design is not reachable for more than two transmit antennas. Weifeng and Xiang-Gen [1] have illustrated that for generalized complex orthogonal designs (square or nonsquare size) without linear processing for more than two transmit antennas the rates cannot be greater than 3/4.

Tarokh et al [3] observed that, complex orthogonal designs (with or without linear processing) need not always be square matrices in order to construct space-time block codes. They also introduced the definition of generalized complex orthogonal designs and they proposed generalized complex orthogonal designs with linear processing. With this new definition, STBC from generalized complex orthogonal design can be developed for any number of transmit antennas. However, for more than six transmit antennas symbol transmission rate is only half which is far from symbol transmission rate one achieved by real orthogonal designs.

STBC from complex orthogonal designs with or without linear processing can be summarized as follows:

- For 2 transmit antennas; STBC exists with the maximum symbol transmission rate 1 from complex orthogonal designs [9].

- For 3 and 4 transmit antennas, STBC exists with symbol transmission rate $3 / 4$ from complex orthogonal designs (with linear processing [3] or without linear processing $[8-10])$.

- For 5 and 6 transmit antennas, STBC exits with symbol transmission rate $3 / 4$ and $3 / 5$, respectively, from complex orthogonal designs with linear processing [10].

- For any number of transmit antennas, STBC exists with symbol transmission rate $1 / 2$ form complex orthogonal designs with linear processing [3].

For two transmit antennas, the Alamouti's coding scheme achieves the maximum possible symbol transmission rate 1 . However, it is not clear what the maximum symbol transmission rate is for more than two transmit antennas when using STBC's from complex orthogonal designs with or without linear processing.

\section{THE CHANNEL MODEL FOR SPACE-TIME BLOCK CODING}

Consider wireless communication system with $n$ transmits antennas and $m$ receives antennas. The path gain $\mathrm{h}(\mathrm{i}, \mathrm{j})$ from transmitter $\mathrm{i}$ to receiver $\mathrm{j}$ such that $1 \leq i \leq n, 1 \leq j \leq m$. Let $l$ be length of block code and transmitted signal at time $t$ by transmitted antenna $\mathrm{i}$ be $s_{t}^{i}, 1 \leq t \leq l, 1 \leq i \leq n$. The received signal at time $t$ by receiver antenna $j$ be $y_{t}^{j}, 1 \leq t \leq$ $l, 1 \leq j \leq m$. Additive white Gaussian noise with mean zero and variance $1 / \mathrm{SNR}$ per dimension be $N_{t}^{j}$. Now, assume quasi static conditions and perfect channel information, that is $\mathrm{h}(\mathrm{i}, \mathrm{j})$ are fixed throughout the block $l$ and change from block to block and are known to the receiver.

A codeword $\mathrm{c}$ in a block code is described by a vector $s=s_{1}^{1}, \ldots, s_{l}^{1}, s_{1}^{2}, \ldots, s_{l}^{2}, \ldots, s_{1}^{n}, \ldots, s_{l}^{n}$. At time $\mathrm{t}$, the receiver obtains

$$
y_{t}^{j}=\sum_{i=1}^{n} h(i, j) s_{t}^{i}+N_{t}^{j}
$$

and select the codeword that minimizes the distance 


$$
\sum_{t=1}^{l} \sum_{j=1}^{m}\left|y_{t}^{j}-\sum_{i=1}^{n} h(i, j) s_{t}^{i}\right|^{2}
$$

\section{REAL ORTHOGONAL DESIGNS}

A real orthogonal design in variable $s_{1}, s_{2}, \ldots, s_{n}$ is an $n x n$ matrix such that entries of orthogonal matrix are $\pm s_{1}, \pm s_{2}, \ldots, \pm s_{n}$. According to [3] an orthogonal design exists for $\mathrm{n}=2,4$ and 8 . By negating some columns of orthogonal matrix one can prepare orthogonal matrix with positive signs to all first row elements. First row of orthogonal matrix can be arranged as $s_{1}, s_{2}, s_{3}, \ldots, s_{n}$ by permuting the columns. Without loss of generality we may assume that orthogonal matrix has this property.

Examples of real orthogonal designs as given in [3] are as follows:

The $2 \times 2$ design

$$
\mathrm{G}_{2}\left(\mathrm{~s}_{1}, \mathrm{~s}_{2}\right)=\left[\begin{array}{rr}
s_{1} & s_{2} \\
-s_{2} & s_{1}
\end{array}\right]
$$

The $4 \times 4$ design

$$
\mathrm{G}_{4}\left(\mathrm{~s}_{1}, \mathrm{~s}_{2}, \mathrm{~s}_{3}, \mathrm{~s}_{4}\right)=\left[\begin{array}{rrrr}
s_{1} & s_{2} & s_{3} & s_{4} \\
-s_{2} & s_{1} & -s_{4} & s_{3} \\
-s_{3} & s_{4} & s_{1} & -s_{2} \\
-s_{4} & -s_{3} & s_{2} & s_{1}
\end{array}\right]
$$

The $8 \times 8$ design

$\mathrm{G}_{8}=\left[\begin{array}{rrrrrrrr}s_{1} & s_{2} & s_{3} & s_{4} & s_{5} & s_{6} & s_{7} & s_{8} \\ -s_{2} & s_{1} & s_{4} & -s_{3} & s_{6} & -s_{5} & -s_{8} & s_{7} \\ -s_{3} & -s_{4} & s_{1} & s_{2} & s_{7} & s_{8} & -s_{5} & -s_{6} \\ -s_{4} & s_{3} & -s_{2} & s_{1} & s_{8} & -s_{7} & s_{6} & -s_{5} \\ -s_{5} & -s_{6} & -s_{7} & -s_{8} & s_{1} & s_{2} & s_{3} & s_{4} \\ -s_{6} & S_{5} & -s_{8} & s_{7} & -s_{2} & s_{1} & -s_{4} & s_{3} \\ -s_{7} & s_{8} & S_{5} & -s_{6} & -s_{3} & s_{4} & s_{1} & -s_{2} \\ -s_{8} & -s_{7} & s_{6} & s_{5} & -s_{4} & -s_{3} & s_{2} & s_{1}\end{array}\right](5)$

All above real orthogonal matrix designs have rate one.

\subsection{Generalized Real Orthogonal Design}

In paper [3], Theorem 4.1.3 presents delay optimal rate one generalized real orthogonal designs for $n=3,5,6,7$ which completes the construction for $n \leq 8$.

$$
\begin{gathered}
G_{3}\left(\mathrm{~s}_{1}, \mathrm{~s}_{2}, \mathrm{~s}_{3}\right)=\left[\begin{array}{cccc}
s_{1} & s_{2} & s_{3} \\
-s_{2} & s_{1} & -s_{4} \\
-s_{3} & s_{4} & s_{1} \\
-s_{4} & -s_{3} & s_{2}
\end{array}\right] \\
G_{5}\left(\mathrm{~s}_{1}, \mathrm{~s}_{2}, \ldots, \mathrm{s}_{8}\right)=\left[\begin{array}{cccccc}
s_{1} & s_{2} & s_{3} & s_{4} & s_{5} \\
-s_{2} & s_{1} & s_{4} & -s_{3} & s_{6} \\
-s_{3} & -s_{4} & s_{1} & s_{2} & s_{7} \\
-s_{4} & s_{3} & -s_{2} & s_{1} & s_{8} \\
-s_{5} & -s_{6} & -s_{7} & -s_{8} & s_{1} \\
-s_{6} & s_{5} & -s_{8} & s_{7} & -s_{2} \\
-s_{7} & s_{8} & s_{5} & -s_{6} & -s_{3} \\
-s_{8} & -s_{7} & s_{6} & s_{5} & -s_{4}
\end{array}\right] \\
G_{6}=\left[\begin{array}{rrrrrr}
\mathrm{s}_{1} & \mathrm{~s}_{2} & \mathrm{~s}_{3} & \mathrm{~s}_{4} & \mathrm{~s}_{5} & \mathrm{~s}_{6} \\
-\mathrm{s}_{2} & \mathrm{~s}_{1} & \mathrm{~s}_{4} & -\mathrm{s}_{3} & \mathrm{~s}_{6} & -\mathrm{s}_{5} \\
-\mathrm{s}_{3} & -\mathrm{s}_{4} & \mathrm{~s}_{1} & \mathrm{~s}_{2} & \mathrm{~s}_{7} & \mathrm{~s}_{8} \\
-\mathrm{s}_{4} & \mathrm{~s}_{3} & -\mathrm{s}_{2} & \mathrm{~s}_{1} & \mathrm{~s}_{8} & -\mathrm{s}_{7} \\
-\mathrm{s}_{5} & -\mathrm{s}_{6} & -\mathrm{s}_{7} & -\mathrm{s}_{8} & \mathrm{~s}_{1} & \mathrm{~s}_{2} \\
-\mathrm{s}_{6} & \mathrm{~s}_{5} & -\mathrm{s}_{8} & \mathrm{~s}_{7} & -\mathrm{s}_{2} & \mathrm{~s}_{1} \\
-\mathrm{s}_{7} & \mathrm{~s}_{8} & \mathrm{~s}_{5} & -\mathrm{s}_{6} & -\mathrm{s}_{3} & \mathrm{~s}_{4} \\
-\mathrm{s}_{8} & -\mathrm{s}_{7} & \mathrm{~s}_{6} & \mathrm{~s}_{5} & -\mathrm{s}_{4} & \mathrm{~s}_{3}
\end{array}\right]
\end{gathered}
$$

$$
G_{7}=\left[\begin{array}{rrrrrrr}
\mathrm{s}_{1} & \mathrm{~s}_{2} & \mathrm{~s}_{3} & \mathrm{~s}_{4} & \mathrm{~s}_{5} & \mathrm{~s}_{6} & \mathrm{~s}_{7} \\
-\mathrm{s}_{2} & \mathrm{~s}_{1} & \mathrm{~s}_{4} & -\mathrm{s}_{3} & \mathrm{~s}_{6} & -\mathrm{s}_{5} & -\mathrm{s}_{8} \\
-\mathrm{s}_{3} & -\mathrm{s}_{4} & \mathrm{~s}_{1} & \mathrm{~s}_{2} & \mathrm{~s}_{7} & \mathrm{~s}_{8} & -\mathrm{s}_{5} \\
-\mathrm{s}_{4} & \mathrm{~s}_{3} & -\mathrm{s}_{2} & \mathrm{~s}_{1} & \mathrm{~s}_{8} & -\mathrm{s}_{7} & \mathrm{~s}_{6} \\
-\mathrm{s}_{5} & -\mathrm{s}_{6} & -\mathrm{s}_{7} & -\mathrm{s}_{8} & \mathrm{~s}_{1} & \mathrm{~s}_{2} & \mathrm{~s}_{3} \\
-\mathrm{s}_{6} & \mathrm{~s}_{5} & -\mathrm{s}_{8} & \mathrm{~s}_{7} & -\mathrm{s}_{2} & \mathrm{~s}_{1} & -\mathrm{s}_{4} \\
-\mathrm{s}_{7} & \mathrm{~s}_{8} & \mathrm{~s}_{5} & -\mathrm{s}_{6} & -\mathrm{s}_{3} & \mathrm{~s}_{4} & \mathrm{~s}_{1} \\
-\mathrm{s}_{8} & -\mathrm{s}_{7} & \mathrm{~s}_{6} & \mathrm{~s}_{5} & -\mathrm{s}_{4} & \mathrm{~s}_{3} & \mathrm{~s}_{2}
\end{array}\right](9)
$$

\section{GENERALIZED COMPLEX ORTHOGONAL DESIGN}

The complex orthogonal designs exit if and only if transmitting antennas are two. Again, allowing linear processing of transmit signals does not increase the set of transmit antennas for which the designs exits. To extend the set of transmitting antennas for complex orthogonal designs, generalized complex orthogonal designs are defined in an analogous fashion as generalized real orthogonal designs. However, block codes are guaranteed to exist for any value of n only for rate $R \leq 1 / 2$.

A complex orthogonal design of size $n$ in variable $s_{1}, s_{2}, \ldots, s_{n}$ is an $\mathrm{n} \times \mathrm{n}$ matrix such that entries of orthogonal matrix are $\pm s_{1}, \pm s_{2}, \ldots, \pm s_{n}$ or their conjugates $\pm s_{1}^{*}, \pm s_{2}^{*}, \ldots, \pm s_{n}^{*}$ or multiples of them by i where $i=\sqrt{-1}$.

\subsection{Alamouti Scheme}

The first STBC from generalized complex orthogonal design was proposed by Alamouti [9] for two transmit antennas. It was a $2 \times 2$ complex orthogonal design in two variables $s_{1}$ and $\mathrm{s}_{2}$ and is given as:

$$
\mathrm{G} 2(\mathrm{~s} 1, \mathrm{~s} 2)=\left[\begin{array}{cc}
s_{1} & s_{2} \\
-s_{2}^{*} & s_{1}^{*}
\end{array}\right]
$$

At the first time slot, s1 and s2 are transmitted by two transmitting antennas simultaneously. At second time slot, second row elements $-s_{2}{ }^{*}$ and $s_{1}{ }^{*}$ are transmitted from two transmitting antennas simultaneously.

\subsection{Generalized Complex Orthogonal Designs Without Linear Processing}

In paper [1], Weifeng and Xiang-Gen showed that for more than two transmit antenna using generalized complex orthogonal designs without linear processing restricts the symbol rate to $3 / 4$.

For three and four transmit antennas, STBC from generalized complex orthogonal designs with rate $\mathrm{R}=3 / 4$ are presented in [4], [11-12] are as follows

$$
\begin{gathered}
\mathrm{G}_{3}\left(\mathrm{~s}_{1}, \mathrm{~s}_{2}, \mathrm{~s}_{3}\right)=\left[\begin{array}{rrr}
S_{1} & S_{2} & S_{3} \\
-S_{2}^{*} & S_{1}^{*} & 0 \\
-S_{3}^{*} & 0 & S_{1}^{*} \\
0 & -S_{3}^{*} & S_{2}^{*}
\end{array}\right] \\
\mathrm{G}_{4}\left(\mathrm{~s}_{1}, \mathrm{~s}_{2}, \mathrm{~s}_{3}\right)=\left[\begin{array}{rrrr}
s_{1} & s_{2} & s_{3} & 0 \\
-s_{2}^{*} & s_{1}^{*} & 0 & s_{3} \\
-s_{3}^{*} & 0 & s_{1}^{*} & -s_{2} \\
0 & -s_{3}^{*} & s_{2}^{*} & s_{1}
\end{array}\right]
\end{gathered}
$$

The fact is, $\mathrm{G}_{3}$ is obtained by taking the first three columns of $\mathrm{G}_{4}$. 


\subsection{Generalized Complex Orthogonal Designs with Linear Processing}

V. Tarokh, H. Jafarkhani and A. R. Calderbank [3] have presented a general design with rate $1 / 2$ for three and four transmit antennas as follows

For four transmit antennas, the design is an $8 \times 3$ matrix

$$
G_{3}\left(s_{1}, s_{2}, s_{3}, s_{4}\right)=\left[\begin{array}{ccc}
s_{1} & s_{2} & s_{3} \\
-s_{2} & s_{1} & -s_{4} \\
-s_{3} & s_{4} & s_{1} \\
-s_{4} & -s_{3} & s_{2} \\
s_{1}^{*} & s_{2}^{*} & s_{3}^{*} \\
-s_{2}^{*} & s_{1}^{*} & -s_{4}^{*} \\
-s_{3}^{*} & s_{4}^{*} & s_{1}^{*} \\
-s_{4}^{*} & -s_{3}^{*} & s_{2}^{*}
\end{array}\right]
$$

For four transmit antennas, the design is an $8 \times 4$ matrix

$$
G_{4}\left(s_{1}, s_{2}, s_{3}, s_{4}\right)=\left[\begin{array}{rrrr}
s_{1} & s_{2} & s_{3} & s_{4} \\
-s_{2} & s_{1} & -s_{4} & s_{3} \\
-s_{3} & s_{4} & s_{1} & -s_{2} \\
-s_{4} & -s_{3} & s_{2} & s_{1} \\
s_{1}^{*} & s_{2}^{*} & s_{3}^{*} & s_{4}^{*} \\
-s_{2}^{*} & s_{1}^{*} & -s_{4}^{*} & s_{3}^{*} \\
-s_{3}^{*} & s_{4}^{*} & s_{1}^{*} & -s_{2}^{*} \\
-s_{4}^{*} & -s_{3}^{*} & s_{2}^{*} & s_{1}^{*}
\end{array}\right]
$$

These transmission schemes for higher $n$ provide full diversity but penalty is they lose half of the theoretical bandwidth efficiency.

In [10], designs of generalized complex orthogonal designs with linear processing of rates higher than $1 / 2$ were presented for 5 and 6 transmit antennas. For five transmit antennas, the design is an $11 \times 5$ matrix given by

$$
G_{5}\left(s_{1}, s_{2}, \cdots, s_{7}\right)\left[\begin{array}{rrrrr}
s_{1} & s_{2} & s_{3} & 0 & s_{4} \\
-s_{2}^{*} & s_{1}^{*} & 0 & s_{3} & s_{5} \\
s_{3}^{*} & 0 & -s_{1}^{*} & s_{2} & s_{6} \\
0 & s_{3}^{*} & -s_{2}^{*} & -s_{1} & s_{7} \\
s_{4}^{*} & 0 & 0 & -s_{7}^{*} & -s_{1}^{*} \\
0 & s_{4}^{*} & 0 & s_{6}^{*} & -s_{2}^{*} \\
0 & 0 & s_{4}^{*} & s_{5}^{*} & -s_{3}^{*} \\
0 & -s_{5}^{*} & s_{6}^{*} & 0 & s_{1} \\
s_{5}^{*} & 0 & s_{7}^{*} & 0 & s_{2} \\
-s_{6}^{*} & -s_{7}^{*} & 0 & 0 & s_{3} \\
s_{7} & -s_{6} & -s_{5} & s_{4} & 0
\end{array}\right]
$$

Actually, $\mathrm{G}_{5}\left(s_{1}, s_{2}, \cdots, s_{7}\right)$ is constructed form $\mathrm{G}_{4}\left(\mathrm{~s}_{1}, \mathrm{~s}_{2}, \mathrm{~s}_{3}\right)$ in equation 12 by considering $\mathrm{G}_{4}$ as $4 \times 4$ sub-matrix of $\mathrm{G}_{5}$ and then symbols $s_{4} s_{5} s_{6} s_{7}$ are added into the fifth column of $G_{5}$. Then entries of $\mathrm{G}_{5}$ from the fifth row to the end are arranged such that all of the 5 columns are orthogonal to each other. Negative signs of G4 are altered in G5 to arrange elements orthogonally. The total number of rows should be as small as possible. The rate of $\mathrm{G}_{5}$ is $\mathrm{R}=7 / 11=0.6364$.

For six transmit antennas, the design will be a $30 \times 6$ matrix as:

$$
G_{6}=\left[\begin{array}{rcrrrr}
s_{1} & s_{2} & s_{3} & 0 & s_{4} & s_{8} \\
-s_{2}^{*} & s_{1}^{*} & 0 & s_{3} & s_{5} & s_{9} \\
s_{3}^{*} & 0 & -s_{1}^{*} & s_{2} & s_{6} & s_{10} \\
0 & s_{3}^{*} & -s_{2}^{*} & -s_{1} & s_{7} & s_{11} \\
s_{4}^{*} & 0 & 0 & -s_{7}^{*} & -s_{1}^{*} & s_{12} \\
0 & s_{4}^{*} & 0 & s_{6}^{*} & -s_{2}^{*} & s_{13} \\
0 & 0 & s_{4}^{*} & s_{5}^{*} & -s_{3}^{*} & s_{14} \\
0 & s_{5}^{*} & -s_{6}^{*} & 0 & -s_{1} & s_{15} \\
s_{5}^{*} & 0 & s_{7}^{*} & 0 & s_{2} & s_{16} \\
s_{6}^{*} & s_{7}^{*} & 0 & 0 & -s_{3} & s_{17} \\
s_{7} & -s_{6} & -s_{5} & s_{4} & 0 & s_{18} \\
s_{8}^{*} & 0 & 0 & -s_{11}^{*} & -s_{15}^{*} & -s_{1}^{*} \\
0 & s_{8}^{*} & 0 & s_{10}^{*} & s_{16}^{*} & -s_{2}^{*} \\
0 & 0 & s_{8}^{*} & s_{9}^{*} & -s_{17}^{*} & -s_{3}^{*} \\
0 & 0 & 0 & s_{18}^{*} & s_{8}^{*} & -s_{4}^{*} \\
0 & 0 & -s_{18}^{*} & 0 & s_{9}^{*} & -s_{5}^{*} \\
0 & -s_{18}^{*} & 0 & 0 & s_{10}^{*} & -s_{6}^{*} \\
s_{18}^{*} & 0 & 0 & 0 & s_{11}^{*} & -s_{7}^{*} \\
0 & -s_{9}^{*} & s_{10}^{*} & 0 & s_{12}^{*} & s_{1} \\
s_{9}^{*} & 0 & s_{11}^{*} & 0 & s_{13}^{*} & s_{2} \\
-s_{10}^{*} & -s_{11}^{*} & 0 & 0 & s_{14}^{*} & s_{3} \\
-s_{12}^{*} & -s_{13}^{*} & -s_{14}^{*} & 0 & 0 & s_{4} \\
-s_{16}^{*} & -s_{15}^{*} & 0 & -s_{14}^{*} & 0 & s_{5} \\
-s_{17}^{*} & 0 & s_{15}^{*} & -s_{13}^{*} & 0 & s_{6} \\
0 & -s_{17}^{*} & -s_{16}^{*} & s_{12}^{*} & 0 & s_{7} \\
0 & s_{14} & -s_{13} & -s_{15} & s_{11} & 0 \\
s_{14} & 0 & -s_{12} & -s_{16} & s_{10} & 0 \\
-s_{13} & -s_{12} & 0 & s_{17} & s_{9} & 0 \\
s_{15} & -s_{16} & s_{17} & 0 & s_{8} & 0 \\
-s_{11} & s_{10} & s_{9} & -s_{8} & s_{18} & 0
\end{array}\right]
$$

\subsection{Complex Orthogonal Design with Rate One}

In [8], L. Xian and H. Liu has proposed codes with rate 1 and codes are based on changing the middle rows of the real codes as

$$
\mathrm{G}_{4}=\left[\begin{array}{rrrr}
s_{1} & s_{2} & s_{3} & s_{4} \\
-s_{2}^{*} & s_{1}^{*} & -s_{4}^{*} & s_{3}^{*} \\
-s_{3}^{*} & s_{4}^{*} & s_{1}^{*} & -s_{2}^{*} \\
-s_{4} & -s_{3} & s_{2} & s_{1}
\end{array}\right]
$$

The above matrix is obtained by real orthogonal design in equation 4 by changing middle half of all rows (second and third) to their complex conjugate.

$$
\mathrm{G}_{8}=\left[\begin{array}{rrrrrrrr}
s_{1} & s_{2} & s_{3} & s_{4} & s_{5} & s_{6} & s_{7} & s_{8} \\
-s_{2} & s_{1} & s_{4} & -s_{3} & s_{6} & -s_{5} & -s_{8} & s_{7} \\
-s_{3}^{*} & -s_{4}^{*} & s_{1}^{*} & s_{2}^{*} & s_{7}^{*} & s_{8}^{*} & -s_{5}^{*} & -s_{6}^{*} \\
-s_{4}^{*} & s_{3}^{*} & -s_{2}^{*} & s_{1}^{*} & s_{8}^{*} & -s_{7}^{*} & s_{6}^{*} & -s_{5}^{*} \\
-s_{5}^{*} & -s_{6}^{*} & -s_{7}^{*} & -s_{8}^{*} & s_{1}^{*} & s_{2}^{*} & s_{3}^{*} & s_{4}^{*} \\
-s_{6}^{*} & s_{5}^{*} & -s_{8}^{*} & s_{7}^{*} & -s_{2}^{*} & s_{1}^{*} & -s_{4}^{*} & s_{3}^{*} \\
-s_{7} & s_{8} & s_{5} & -s_{6} & -s_{3} & s_{4} & s_{1} & -s_{2} \\
-s_{8} & -s_{7} & s_{6} & s_{5} & -s_{4} & -s_{3} & s_{2} & s_{1}
\end{array}\right]
$$

The above matrix in equation 18 is obtained by real orthogonal design in equation 5 by changing middle half of all rows (second and third) to their complex conjugate.

\section{CONCLUSION}

The goal of this paper was to present an overview of real and complex orthogonal designs in space-time block coding used for wireless communication system. Generalized real and complex orthogonal designs were discussed here. Designs with rate one complex orthogonal design were also elaborated on. 
Future scope includes the extension of this work with higher rate codes.

\section{REFERENCES}

[1] Weifeng Su and Xiang-Gen Xia. 2003. "On Space-Time Block Codes from Complex Orthogonal Designs", Wireless Personal Communications 25: 1-26.

[2] A.V. Geramita and J. Seberry. 1979. Orthogonal Designs, Quadratic Forms and Hadamard Matrices. Lecture Notes in Pure and Applied Mathematics, Vol. 43, Marcel Dekker: New York and Basel.

[3] V. Tarokh, H. Jafarkhani and A.R. Calderbank. 1999. "Space-time Block Codes from Orthogonal Designs", IEEE Trans. Inform. Theory, Vol. 45, No. 5, pp. 14561467.

[4] G. Ganesan and P. Stoica. 2001. "Space-time Block Codes: A Maximum SNR Approach", IEEE Trans. Inform. Theory, Vol. 47, No. 4, pp. 1650-1656.

[5] Seberry, J., Spence, S. A., \& Wysocki, T. A. 2005. A construction technique for generalized complex orthogonal designs and applications to wireless communications. Linear algebra and its applications, 405, 163-176.
[6] T. Josefiak. 1976. "Realization of Hurwitz-Radon Matrices", Queen's Papers in Pure and Applied Mathematics, No. 36, pp. 346-351.

[7] W. Wolfe. 1976. "Amicable Orthogonal Designs Existence”, Canad. J. Math., Vol. 28, pp. 1006-1020.

[8] Liang Xian and Huaping Liu. 2005. "Rate-One SpaceTime Block Codes With Full Diversity", IEEE Trans. Commun, Vol. 53, No. 12.

[9] S. Alamouti. 1998. "A Simple Transmit Diversity Technique forWireless Communications", IEEE J. Select. Areas Commun., Vol. 16, No. 8, pp. 1451-1458.

[10] Su, W., \& Xia, X. G. 2003. Two generalized complex orthogonal space-time block codes of rates $7 / 11$ and $3 / 5$ for 5 and 6 transmit antennas. Information Theory, IEEE Transactions on, 49(1), 313-316.

[11] O. Tirkkonen and A. Hottinen. 2002 "Square-matrix Embeddable Space-time Block Codes for Complex Signal Constellations", IEEE Trans. Inform. Theory, Vol. 48, No. 2, pp. 1122-1126.

[12] B.M. Hochwald, T.L. Marzetta and C.B. Papadias. 2001 "A Transmitter Diversity Scheme for Wideband CDMA Systems Based on Space-time Spreading", IEEE J. Select. Areas Commun., Vol. 19, No. 1, pp. 48-60. 